\title{
Completion of annual diabetes care processes and mortality: A cohort study using the National Diabetes Audit for England and Wales
}

\author{
Naomi Holman PhD ${ }^{1}$ | Peter Knighton MPhys ${ }^{2}$ | Jackie O'Keefe MSc ${ }^{2}$ | \\ Sarah H. Wild PhD ${ }^{3}$ | Sarah Brewster MRCP ${ }^{4}$ | Hermione Price FRCP ${ }^{4}$ | | \\ Kiran Patel PhD $5,6,7$ | Wasim Hanif MD ${ }^{8}$ | Vinod Patel MD ${ }^{6,9,10 ~ \mid ~}$ \\ Edward W. Gregg PhD ${ }^{11}$ \\ Kamlesh Khunti MD ${ }^{13}$ \\ Richard I. G. Holt PhD ${ }^{12}$ \\ Roger Gadsby $\mathrm{MB}^{6}$ \\ Naveed Sattar MD ${ }^{1}$ (1) \\ ${ }^{1}$ Institute of Cardiovascular and Medical Sciences, University of Glasgow, Glasgow, UK \\ ${ }^{2}$ Analytical Services - Population Health, Clinical Audit and Specialist Care, NHS Digital, Leeds, UK \\ ${ }^{3}$ College of Medicine and Veterinary Medicine, Usher Institute, University of Edinburgh, Edinburgh, UK \\ ${ }^{4}$ West Hampshire Community Diabetes Service, Southern Health NHS Foundation Trust, Lyndhurst, UK \\ ${ }^{5}$ University Hospitals Coventry and Warwickshire NHS Trust, Coventry, UK \\ ${ }^{6}$ Warwick Medical School, University of Warwick, Warwick, UK \\ ${ }^{7}$ Coventry University, Coventry, UK \\ ${ }^{8}$ University Hospitals Birmingham NHS Foundation Trust, Birmingham, UK \\ ${ }^{9}$ Diabetes and Endocrinology Centre, George Eliot Hospital NHS Trust, Nuneaton, UK \\ ${ }^{10}$ West Midlands Clinical Networks and Clinical Senate, NHS England and NHS Improvement - Midlands, Birmingham, UK \\ ${ }^{11}$ School of Public Health, Imperial College, London, UK \\ ${ }^{12}$ Human Development and Health, Faculty of Medicine, University of Southampton and Southampton National Institute for Health Research Biomedical Research \\ Centre, University Hospital Southampton NHS Foundation Trust, Southampton, UK \\ ${ }^{13}$ Diabetes Research Centre, University of Leicester, Leicester, UK \\ ${ }^{14} \mathrm{NHS}$ England and NHS Improvement, London, UK \\ ${ }^{15}$ Department of Diabetes and Endocrinology, St Mary's Hospital, Imperial College Healthcare NHS Trust, London, UK \\ ${ }^{16}$ Division of Metabolism, Digestion and Reproduction, Imperial College London, London, UK \\ ${ }^{17}$ Diabetes UK, London, UK
}

\section{Correspondence}

Dr Naomi Holman, PhD, c/o RC214 Level C2, Institute of Cardiovascular and Medical Sciences, BHF Glasgow Cardiovascular Research Centre, University of Glasgow, 126 University Place, Glasgow G12 8TA, UK.

Email: naomi.holman@glasgow.ac.uk

Funding information

British Heart Foundation, Grant/Award

Number: RE/18/6/34217; Diabetes UK; NHS

England and NHS Improvement; South Asian

Health Foundation

\begin{abstract}
Aim: To conduct an analysis to assess whether the completion of recommended diabetes care processes (glycated haemoglobin [HbA1c], creatinine, cholesterol, blood pressure, body mass index [BMI], smoking habit, urinary albumin, retinal and foot examinations) at least annually is associated with mortality.

Materials and methods: A cohort from the National Diabetes Audit of England and Wales comprising 179105 people with type 1 and 1397790 people with type 2 diabetes, aged 17 to 99 years on January 1, 2009, diagnosed before
\end{abstract}


January 1, 2009 and alive on April 1, 2013 was followed to December 31, 2019. Cox proportional hazards models adjusting for demographic characteristics, smoking, HbA1c, blood pressure, serum cholesterol, BMI, duration of diagnosis, estimated glomerular filtration rate, prior myocardial infarction, stroke, heart failure, respiratory disease and cancer, were used to investigate whether care processes recorded January 1, 2009 to March 31, 2010 were associated with subsequent mortality.

Results: Over a mean follow-up of 7.5 and 7.0 years there were 26915 and 388093 deaths in people with type 1 and type 2 diabetes, respectively. Completion of five or fewer, compared to eight, care processes (retinal screening not included as data were not reliable) had a mortality hazard ratio (HR) of 1.37 (95\% confidence interval [Cl] 1.28-1.46) in people with type 1 and 1.32 (95\% Cl 1.30-1.35) in people with type 2 diabetes. The HR was higher for respiratory disease deaths and lower in South Asian ethnic groups.

Conclusions: People with diabetes who have fewer routine care processes have higher mortality. Further research is required into whether different approaches to care might improve outcomes for this high-risk group.

\section{KEYWORDS}

cohort study, type 1 diabetes, type 2 diabetes

\section{1 | INTRODUCTION}

Optimal management of blood glucose, lipids and blood pressure reduces the microvascular and macrovascular complications of diabetes. ${ }^{1-3}$ Accordingly, measurement and management of glycated haemoglobin $(\mathrm{HbA} 1 \mathrm{c})$, blood pressure and lipid profile are at the centre of national and international diabetes care guidelines. ${ }^{4-7}$ Regular review of these and other risk factors for complications, including weight and smoking habit, are recommended, as are tests for early detection of kidney, foot and eye disease.

In England, the National Institute for Health and Care Excellence (NICE) recommends that people with type 1 diabetes ${ }^{4}$ and type 2 diabetes $^{5}$ are offered nine annual processes (measurement of $\mathrm{HbA} 1 \mathrm{c}$, lipids, creatinine, albuminuria, blood pressure and body mass index [BMI], ascertainment of smoking status, and examination of the feet and retinae), and the completion of these has been incentivized in primary care. ${ }^{8}$ Most international guidelines also stress the importance of these care processes. However, whilst their regular completion might seem intuitively sensible, the level of evidence to support the guideline- recommended processes, including their effect on clinical outcomes, is usually not known or is rated at the lowest standard of evidence ("expert consensus" or "clinical experience"). $^{7}$

In England and Wales, the National Diabetes Audit (NDA) collects patient-level data on people with diagnosed diabetes. The present study assesses whether recorded care processes completion was associated with mortality over the subsequent decade after adjustment for the risk factors that the care processes uncover, individual demographic characteristics and comorbidities.

\section{2 | MATERIALS AND METHODS}

\section{$2.1 \quad$ Data sources}

The NDA has collated data on people with diagnosed diabetes registered with a primary or specialist healthcare provider in England since 2003. Individuals receiving care from general practice and specialist outpatient services based in acute and community trusts are included if they have a valid code for diabetes mellitus (excluding gestational diabetes) in their electronic health record. ${ }^{9}$ The 2009/2010 NDA data collection included data from 6700 (76\%) general practices and was estimated to include data on $81.1 \%$ people aged 17 years and older with diagnosed diabetes in England and Wales. ${ }^{10}$

These data were linked to Hospital Episode Statistics and the Patient Episode Database for Wales, which records all hospital admissions in England and Wales, respectively, and to civil death registrations in both countries collated by the Office for National Statistics.

The legal basis for the NDA data collection and linkage is a "direction" from NHS England to NHS Digital according to section 254 of the Health and Social Care Act for England 2012; in Wales it is granted under section 270 of the Health and Social Care Act. To protect confidentiality, all data with a final digit of $1,2,8$ or 9 are 
rounded to 0 , and 3, 4, 6 or 7 are rounded to 5 . Numbers with a final digit of 0 or 5 are unchanged.

\section{2 | Study population and observation period}

The study population was people aged between 17 and 99 years on January 1, 2009, diagnosed with type 1 diabetes and type 2 diabetes before January 1, 2009 who were included in the 2009/2010 NDA data collection and still alive on April 1, 2013. Analysis was restricted to individuals who survived 3 years after the exposure period to reduce potential bias from the clinically appropriate suspension of diabetes care processes for people in end-of-life care. Individuals were followed up from April 1, 2013 until death or December 31, 2019.

\section{3 | Outcomes}

The outcomes were death from all causes and underlying (primary) cause of death from cardiovascular disease (International Classification of Disease [ICD]-10 codes I01-199), cancer (ICD-10 codes C01-C99), respiratory disease (ICD-10 codes J01-J99), diabetesspecific causes (ICD-10 codes E10-14) and renal disease (ICD-10 codes N17-19).

\section{$2.4 \mid$ Exposures}

Data secondarily recorded in general practice systems for retinal examinations for this period are not considered reliable. The primary exposure was, therefore, the number out of a total of eight care processes (blood tests for $\mathrm{HbA1c}$, cholesterol, creatinine, measurement of blood pressure, BMI, albuminuria, smoking habit assessment and the examination of feet) recorded as undertaken between January 1, 2009 and March 31, 2010. As initial exploratory analysis identified that only a minority of people had five or fewer care processes recorded and that people receiving six or seven care processes had similar characteristics and outcomes, these categories were used in the analysis. People who had all eight care processes recorded formed the primary reference group to reflect current national guidelines.

Age and duration of diagnosed diabetes at baseline were calculated using date of birth and date of diagnosis, respectively. Ethnicity was based on self-reported ethnic group as recorded by healthcare providers and classified as White, Mixed, South Asian, Black, other or missing. Type of diabetes was attributed based on the most recent type recorded by a healthcare provider and notified to the NDA. Data from a specialist healthcare provider were assigned precedence over the type of diabetes in the primary care health record.

Deprivation was measured using the area-based Index of Multiple Deprivation $2007^{11}$ based on the home postcode recorded in the $2009 / 2010$ NDA data collection and split into quintiles for analysis.

The latest reported risk factor measurements in the period January 1, 2009 to March 31, 2010 for HbA1c, systolic blood pressure, total cholesterol, creatinine, BMI and smoking habit were identified. Estimated glomerular filtration rate (eGFR) was calculated using the Modification of Diet in Renal Disease formula. ${ }^{12}$

Hospital admissions for myocardial infarction (ICD-10 codes 121-22), stroke (ICD-10 codes 161, 163-64, 167.9), heart failure (ICD-10 codes I50), respiratory disease (ICD-10 codes J01-99) and cancer (ICD-10 codes C01-99) between January 1, 2004 and December 31, 2008 were identified.

\section{5 | Statistical methods}

The differences in mean age, duration of diagnosed diabetes, $\mathrm{HbA} 1 \mathrm{c}$ and $\mathrm{BMI}$ by the number of care processes recorded as undertaken were tested using analysis of variance (ANOVA), with Levene's test to identify differences in variance. Differences in the proportion of people recorded as receiving care processes for categorical variables (sex, social deprivation, ethnicity, smoking habit) were tested using the chi-squared statistic. Crude mortality rates and mortality rates per 1000 person-years, standardized for age and sex to the European Standard population, were calculated with 95\% confidence intervals (Cls) using Byar's method. ${ }^{13}$

Cox proportional hazard models were created to assess the associations between the number of recorded care processes and mortality for people with type 1 and people with type 2 diabetes. A series of models was created consisting of sequentially more covariates to examine potential confounding factors.

Separate models, adjusting for all risk factors, were created for mortality from cardiovascular disease, cancer, respiratory disease, diabetes-specific causes and renal failure for type 1 diabetes and type 2 diabetes separately. Models adjusted for all risk factors and stratified by sex, age (less than 65 years old and 65 years and older), ethnic group, quintile of deprivation and whether or not the individual had an acute hospital admission in the year prior to the exposure period were constructed for all-cause mortality in people with type 1 diabetes and in people with type 2 diabetes.

Two models (one for type 1 diabetes and one for type 2 diabetes) adjusted for age, sex, ethnic group, deprivation and whether or not each of the eight care processes had been completed were created to identify if the association with all-cause mortality varied by type of care process. All variables were defined as categorical variables and included a category for missing data. A sensitivity analysis was undertaken in which everyone included in the 2009/2010 NDA and still alive on January 1, 2011 to explore whether the survival bias introduced by excluding deaths shortly after the exposure period altered the findings.

Statistical analysis was undertaken in SAS Enterprise Guide 7.1.

\section{3 | RESULTS}

A total of 179105 people with type 1 diabetes and 1397790 with type 2 diabetes were followed up for a mean (SD) of 7.5 (1.4) and 7.0 (1.8) years, respectively. Among those with type 1 diabetes, 80635 (45.0\%) had received all eight care processes at least once between January 1, 2009 and March 31, 2010, 61230 (34.2\%) had received six or seven care 


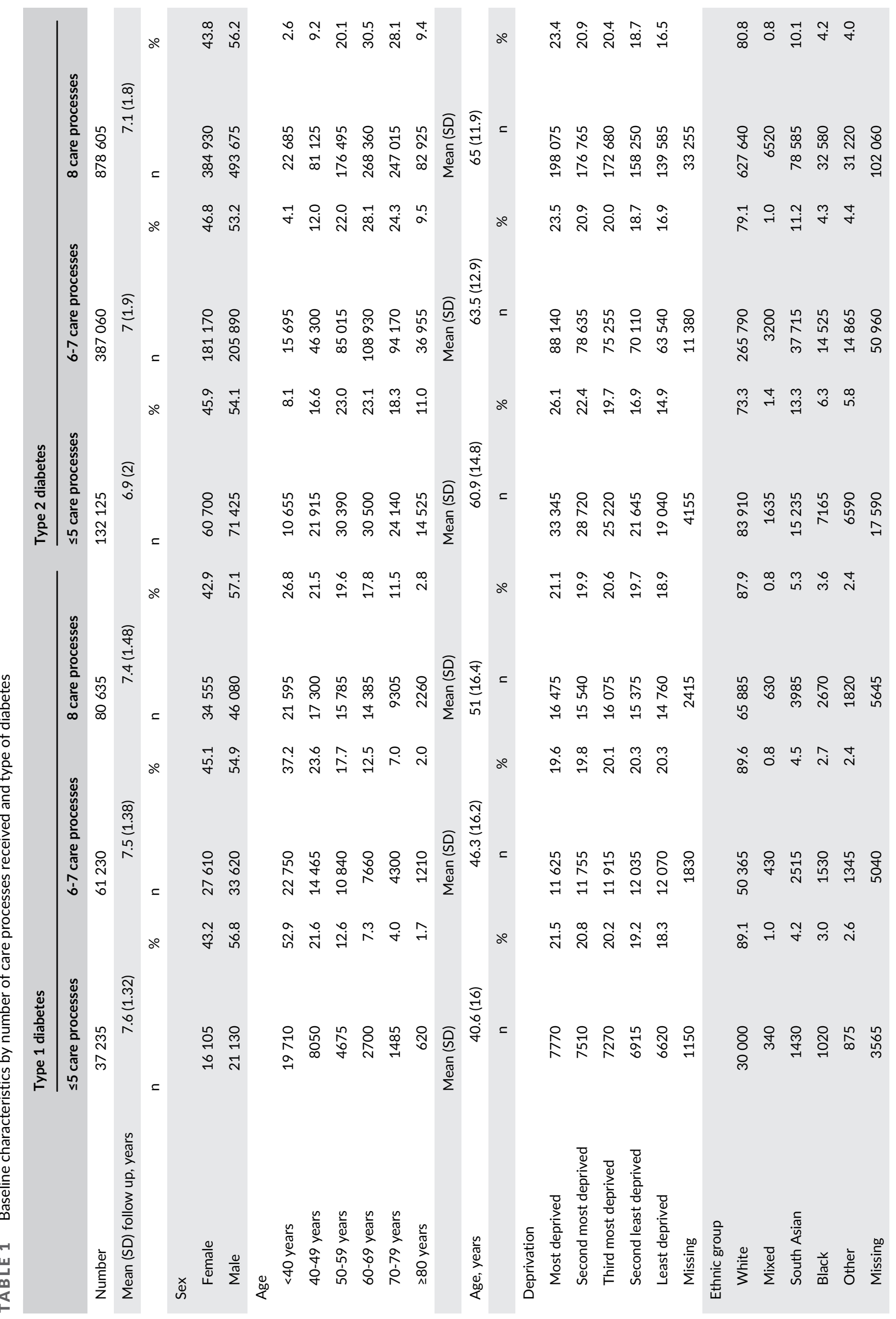




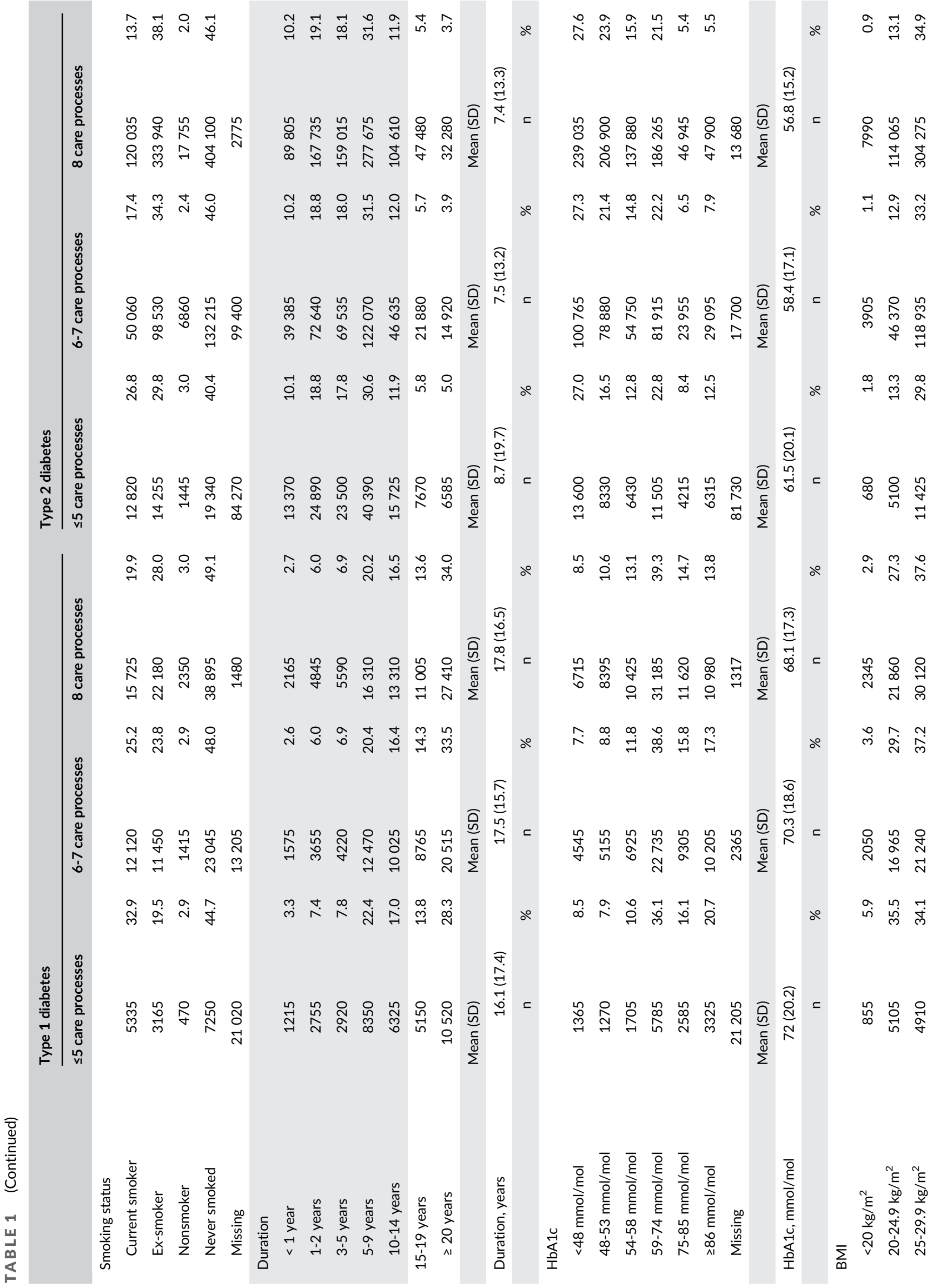




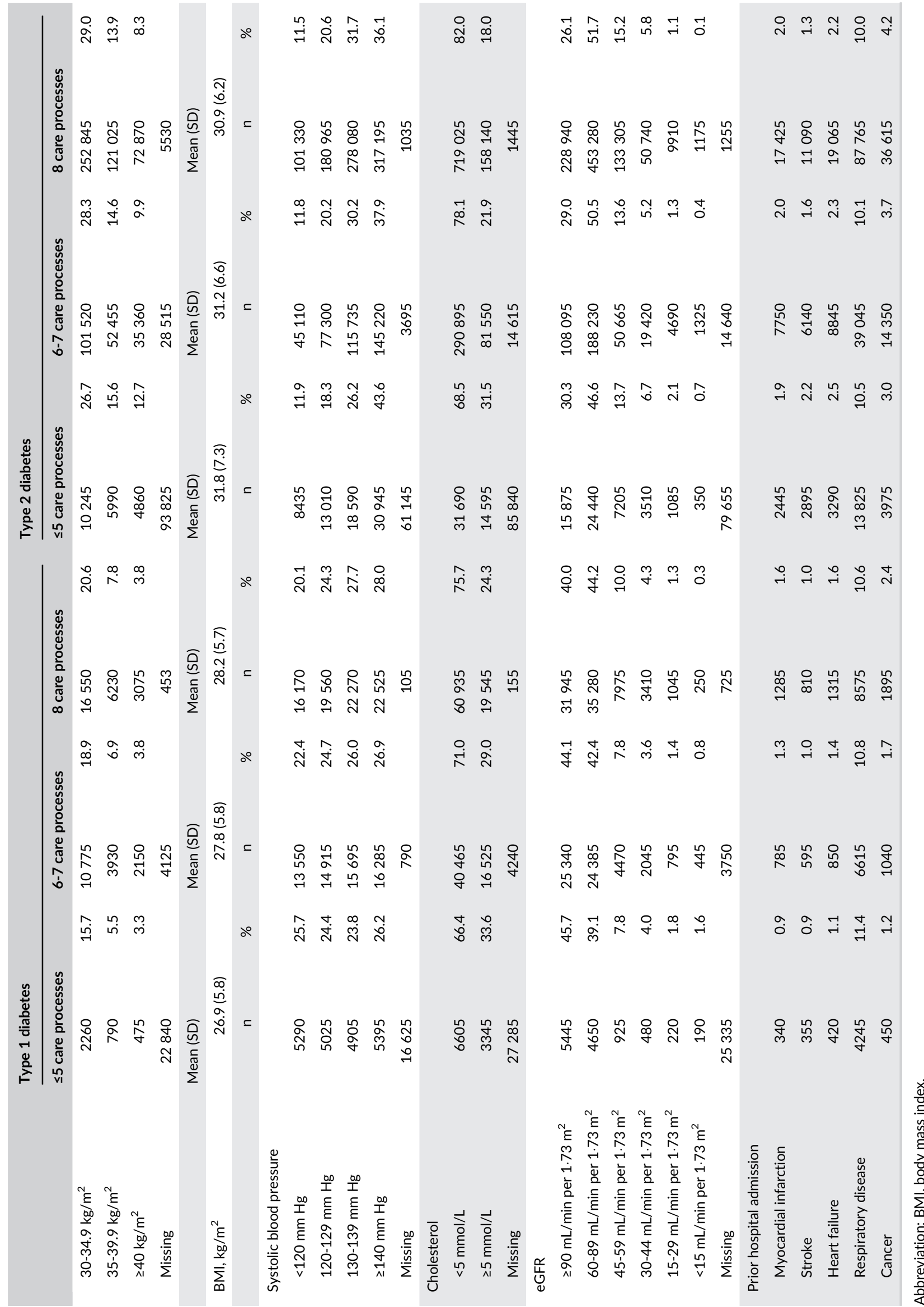




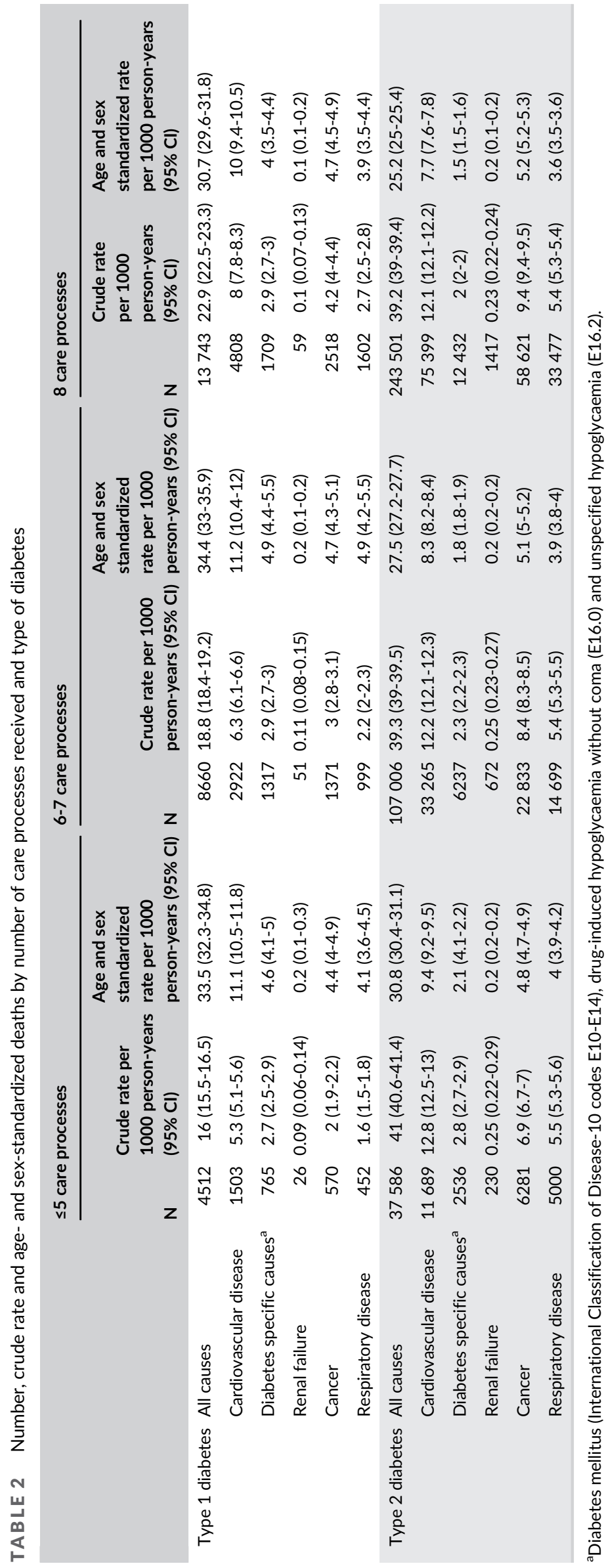


processes, whilst 37235 (20.8\%) had received five or fewer care processes in the same period. The corresponding figures for people with type 2 diabetes were 878605 (62.9\%), 387060 (27.6\%) and 132125 (9.5\%), respectively.

\subsection{Characteristics by number of care processes received}

Care process completion variation showed little relation to deprivation but was associated with age, ethnicity, $\mathrm{HbA1c}$ and smoking status (Table 1). The mean age of those with type 1 diabetes recorded as having received five or fewer care processes was 40.6 years compared to mean ages of 46.3 and 51.0 years for those recorded as receiving six or seven care processes and all eight recommended care processes, respectively $(P<0.005)$. For those with type 2 diabetes, the mean ages were $60.9,63.5$ and 65.0 years, respectively $(P<0.005)$. A total of $97.6 \%$ of those with type 1 and $97.6 \%$ of those with type 2 diabetes had a valid ethnic group recorded. Among those with type 1 diabetes, $89.1 \%$ of those recorded as receiving five or fewer care processes and $87.9 \%$ recorded as receiving all eight care processes were from White ethnic groups $(P<0.005)$; the corresponding proportions in those with type 2 diabetes were $73.3 \%$ and $80.8 \%(P<0.005)$. The latest mean $\mathrm{HbA1c}$ recorded between January 1, 2009 and March 31, 2010 was higher in those recorded as receiving fewer care processes: in people with type 1 diabetes, it was $72 \mathrm{mmol} / \mathrm{mol}$ (8.7\%) for five or fewer, $70.3 \mathrm{mmol} / \mathrm{mol}$ (8.6\%) for six or seven compared to $68 \mathrm{mmol} / \mathrm{mol}(8.4 \%)$ for eight care processes $(P<0.005)$, and in those with type 2 diabetes, it was $62 \mathrm{mmol} / \mathrm{mol}$ (7.8\%) for five or fewer, $58.4 \mathrm{mmol} / \mathrm{mol}$ (7.5\%) for six or seven and $57 \mathrm{mmol} / \mathrm{mol}$ (7.4\%) for eight care processes $(P<0.005)$. Smoking prevalence recorded between January 1, 2009 and March 31, 2010 was higher among those receiving fewer care processes: $32.9 \%$ for five or fewer versus $19.9 \%$ for eight care processes in type 1 diabetes $(P<0.005)$ and $26.8 \%$ versus $13.7 \%$ in type 2 diabetes $(P<0.005)$.

A breakdown of the individual care processes received is provided in Tables S1 and S2.

\section{2 | Mortality by number of care processes received}

Over the period January 1, 2012 to December 31, 2019 there were 26915 deaths over 1431940 person-years of follow-up in people with type 1 diabetes and 388093 deaths over 9853914 person-years of follow-up in those with type 2 diabetes. The all-cause age- and sexstandardized mortality rate for people with type 1 diabetes with five or fewer care processes was 33.5 per 1000 person years $(95 \% \mathrm{Cl} 32.3-$ 34.8), compared to 34.4 ( $95 \% \mathrm{Cl} 33.5-35.9$ ) for those with six or seven care processes recorded and 30.7 (95\% Cl 29.6-31.8) for those with eight care processes recorded. The corresponding figures for people with type 2 diabetes were $30.8(95 \% \mathrm{Cl} 30.4-31.1), 27.5(95 \% \mathrm{Cl}$ 27.2-27.7) and 25.2 (95\% Cl 25.0-25.4; Table 2).
After adjustment for age, sex, ethnicity and deprivation, five or fewer processes recorded and having six or seven care processes recorded during the period January 1, 2009 to March 31, 2010 was inversely associated with higher all-cause mortality (hazard ratio [HR] compared to eight care processes recorded $1.17[95 \% \mathrm{Cl}$ 1.14-1.20] for six or seven, 1.35 [95\% Cl 1.29-1.41] for five or fewer in type 1 diabetes and 1.15 [95\% Cl 1.14-1.16] for six or seven, 1.36 [95\% Cl 1.34-1.38] for five or fewer in type 2 diabetes). Further adjustment to include smoking habit, $\mathrm{HbA1c}$, systolic blood pressure, serum cholesterol, BMI and duration of diagnosed diabetes increased the HR for all-cause mortality associated with having five or fewer care processes to 1.38 (95\% Cl 1.29-1.47) for type 1 diabetes, and decreased it to 1.33 (95\% Cl 1.30-1.35) for type 2 diabetes. Adding in eGFR and prior hospital admissions for myocardial infarction, stroke, heart failure, respiratory disease and cancer slightly attenuated these HRs (Table 3).

After adjustment for all covariates, the gradient of the inverse association of mortality in people with type 2 diabetes with number of recorded care processes was lower for cancer deaths (Table 3). In contrast, the gradient for respiratory disease deaths was higher: HRs of 1.45 (95\% Cl 1.19-1.76) in type 1 diabetes and 1.41 (95\% Cl 1.33-1.49) in type 2 diabetes for those with five or fewer care processes compared to those with eight care processes recorded.

Among people with type 2 diabetes the inverse association between recorded care processes completion was steeper in women than men (HR for five or fewer compared to eight care processes 1.36 [95\% Cl 1.32-1.40] for women compared to 1.29 [95\% Cl 1.25-1.33] for men; Figure 1B). The HRs for death associated with different numbers of recorded care processes were similar in people aged under or over 65 years in both type 1 diabetes and type 2 diabetes (Figure 1A,B).

In people with type 2 diabetes the HRs for death associated with the number of recorded care processes were similar in White and Black ethnic groups but significantly lower in South Asian ethnic groups (Figure 1). In people with type 1 diabetes, the Cls were much broader and no differences between ethnic groups were identified. In both type 1 diabetes and type 2 diabetes the HRs associated with numbers of recorded care processes were similar across all deprivation quintiles (Table S3). For people who had one or more acute hospital admission in the year prior to the exposure period the all-cause mortality HR associated with receiving fewer than five care processes was lower than for those who did not have an acute hospital admission (1.29 [95\% Cl 1.14-1.45] compared to 1.36 [95\% Cl 1.26-1.47] in type 1 diabetes and 1.27 [95\% Cl 1.21-1.32] compared to 1.32 [95\% Cl 1.29-1.35] in type 2 diabetes).

\section{3 | Individual care processes}

Associations adjusted for age, sex, ethnicity and deprivation were investigated according to individual care process (Table S4). Not having BMI measured was associated with the greatest HR for all-cause mortality (1.36 [95\% Cl 1.30-1.43] for type 1 diabetes and 1.40 [95\% Cl 1.38-1.42] for type 2 diabetes), followed by not having a 
TAB LE 3 Hazard ratios for mortality associated with the number of care processes recorded between January 1, 2009 and March 31, 2010 for people with type 1 diabetes and type 2 diabetes, all-cause mortality with different adjustments and cause-specific mortality

\begin{tabular}{|c|c|c|c|}
\hline Cause of death & Care processes received & Type 1 diabetes HR (95\% Cl) & Type 2 diabetes HR $(95 \% \mathrm{Cl})$ \\
\hline \multirow[t]{2}{*}{ All causes ${ }^{a}$} & $\leq 5$ & $1.35(1.29-1.41)$ & $1.36(1.34-1.38)$ \\
\hline & 6 or 7 & $1.17(1.14-1.2)$ & $1.15(1.14-1.16)$ \\
\hline \multirow[t]{2}{*}{ All causes ${ }^{b}$} & $\leq 5$ & $1.38(1.29-1.47)$ & $1.33(1.3-1.35)$ \\
\hline & 6 or 7 & $1.12(1.09-1.16)$ & $1.1(1.09-1.11)$ \\
\hline \multirow[t]{3}{*}{ All causes ${ }^{c}$} & $\leq 5$ & $1.37(1.28-1.46)$ & $1.32(1.3-1.35)$ \\
\hline & 6 or 7 & $1.11(1.08-1.14)$ & $1.1(1.09-1.11)$ \\
\hline & All 8 & 1.00 & 1.00 \\
\hline \multirow[t]{2}{*}{ Cardiovascular disease $^{c}$} & $\leq 5$ & $1.32(1.18-1.48)$ & $1.28(1.24-1.33)$ \\
\hline & 6 or 7 & $1.06(1.01-1.11)$ & $1.09(1.07-1.1)$ \\
\hline Cancer $^{\mathrm{c}}$ & All 8 & 1.00 & 1.00 \\
\hline \multirow[t]{3}{*}{ Respiratory disease $^{c}$} & $\leq 5$ & $1.45(1.19-1.76)$ & $1.41(1.33-1.49)$ \\
\hline & 6 or 7 & $1.19(1.1-1.3)$ & $1.14(1.12-1.17)$ \\
\hline & All 8 & 1.00 & 1.00 \\
\hline \multirow[t]{3}{*}{ Diabetes-specific causes $^{c}$} & $\leq 5$ & $1.16(0.98-1.36)$ & $1.37(1.26-1.49)$ \\
\hline & 6 or 7 & $1.15(1.06-1.24)$ & $1.18(1.14-1.22)$ \\
\hline & All 8 & 1.00 & 1.00 \\
\hline Renal failure $^{c}$ & $\leq 5$ & $1.52(0.66-3.51)$ & $1.27(0.98-1.66)$ \\
\hline
\end{tabular}

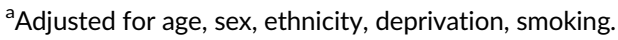

${ }^{\mathrm{b}}$ Adjusted for age, sex, ethnicity, deprivation, smoking, glycated haemoglobin (HbA1c), systolic blood pressure, cholesterol, body mass index (BMI), duration of diagnosis.

${ }^{c}$ Adjusted for age, sex, ethnicity, deprivation, smoking, $\mathrm{HbA1c}$, systolic blood pressure, cholesterol, BMI, durations of diagnosis, estimated glomerular filtration rate, prior hospital admission for myocardial infarction, stroke, heart failure, respiratory disease and cancer.

Abbreviation: $\mathrm{Cl}$, confidence interval; $\mathrm{HR}$, hazard ratio.

cholesterol measurement (1.21 [95\% Cl 1.14-1.28] for type 1 diabetes and 1.22 [95\% $\mathrm{Cl} 1.20-1.25]$ for type 2 diabetes). By contrast, for both type 1 diabetes and type 2 diabetes no record of blood pressure $(0.64$ [95\% Cl 0.60-0.69]; 0.67 [95\% Cl 0.65-0.68]), smoking status (0.86 [95\% Cl 0.83-0.89]; 0.91 [95\% Cl 0.90-0.92]) or serum creatinine (0.66 [95\% Cl 0.62-0.71]; 0.82 [95\% Cl 0.80-0.84]) were associated with lower mortality hazards. Not having an $\mathrm{HbA1c}$ measurement recorded was associated with higher all-cause mortality in type 1 diabetes (HR 1.24 [95\% Cl 1.16-1.33]) but with lower mortality in type 2 diabetes (HR 0.91 [95\% $\mathrm{Cl} 0.89-0.93])$.

\section{4 | DISCUSSION}

This large national population-based cohort of people with type 1 diabetes and type 2 diabetes followed up for means of 7.6 and 6.9 years, respectively, following 15 months of routine care, finds that having five or fewer recorded care processes during that baseline period was associated with subsequent 7-year hazards of all-cause mortality approximately one-third higher compared to having all eight care processes after accounting for demographic characteristics. This higher mortality persists after adjustment for clinical factors known to affect the risk of diabetes-related complications ( $\mathrm{HbA1c}$, systolic blood pressure, serum cholesterol, BMI, smoking habit), and cardiovascular and renal comorbidities were taken into account.

The associations were similar between people with type 1 diabetes and type 2 diabetes, at all ages and across socioeconomic groups. In England and Wales most people with type 1 diabetes have specialist-led care while, for type 2 diabetes, most people are managed in a primary care setting. ${ }^{14}$ Accordingly, the association between the number of recorded care processes and mortality was independent of the type of care setting. During periods of acute illness or palliative care the medium- to long-term management of diabetes-associated risk may not have clinical priority. 
(A)

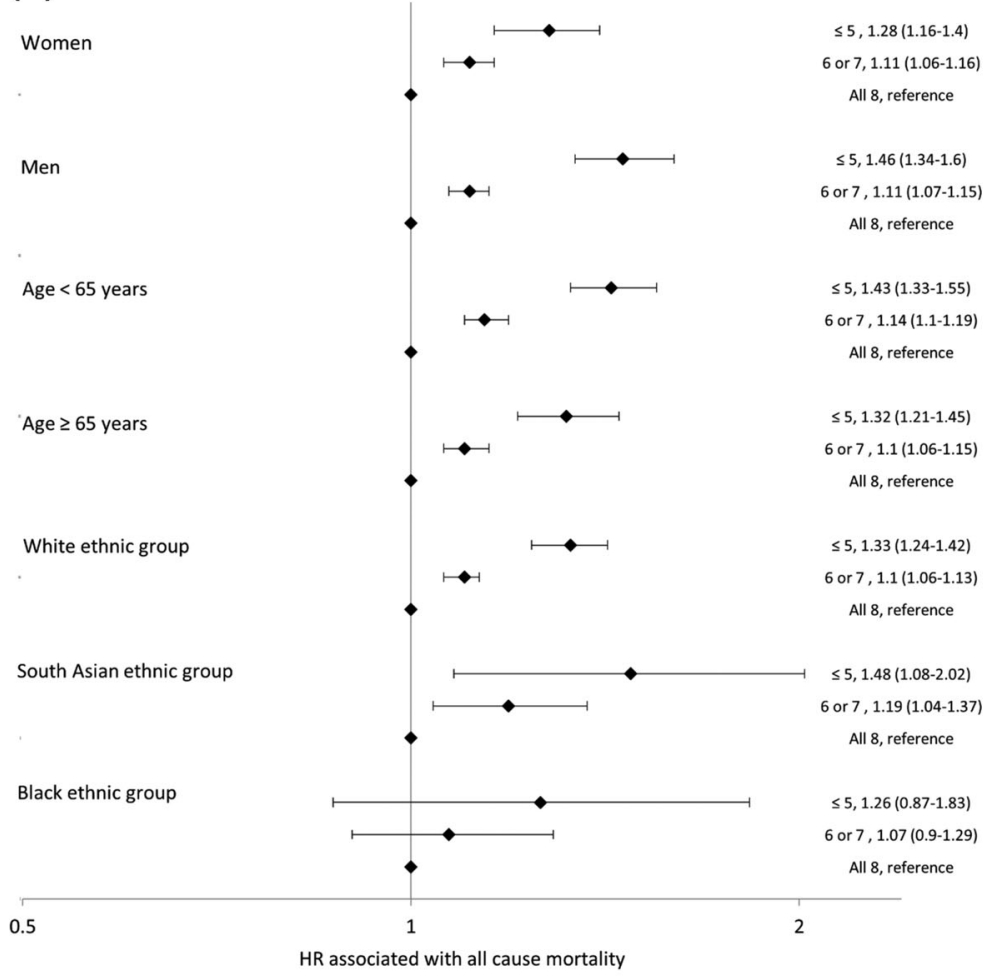

Adjusted for age, sex, social deprivation, ethnicity, smoking, duration of diagnosed diabtes, HbA1c, systolic blood pressure, body mass index eGFR, prior hospital admission for MI, stroke, heart failure, respiratory disease and cancer

(B)

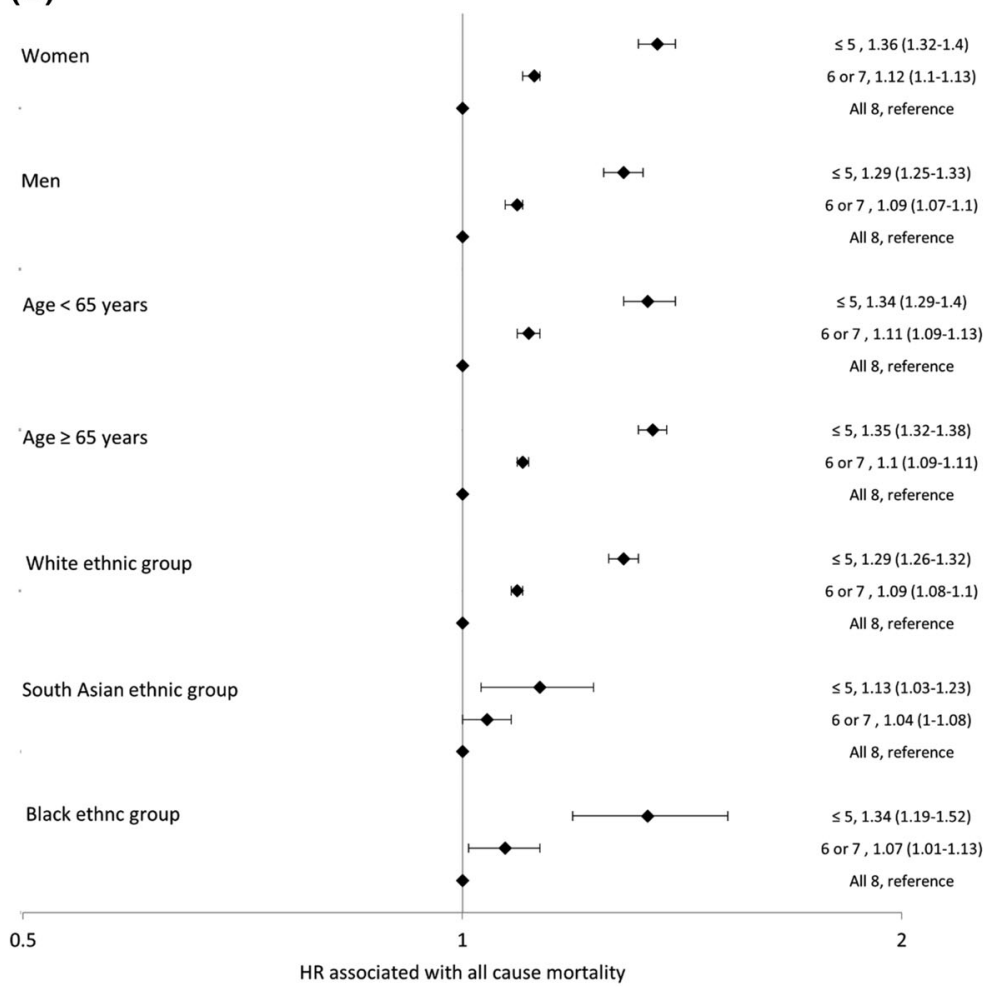

Adjusted for age, sex, social deprivation, ethnicity, smoking, duration of diagnosed diabetes, HbA1c, systolic blood pressure, body mass index eGFR, prior hospital admission for MI, stroke, heart failure, respiratory disease and cancer

FIGURE 1 A, Forest plot of the hazard ratio (HR) for all-cause mortality associated with number of care processes recorded between January 1, 2009 and March 31, 2010, stratified by sex, age and ethnicity for people with type 1 diabetes. B, Forest plot of HR for all-cause mortality associated with number of care processes recorded between January 1, 2009 and March 31, 2010 stratified by sex, age and ethnicity for people with type 2 diabetes 
Nonetheless, the association of higher mortality persists in people who had one or more acute hospital admission in the year prior to the assessment of care processes, although the HRs for this group are lower than for those without an acute hospital admission, perhaps reflecting a partial de-prioritization of routine diabetes care at times of acute illness. This finding, combined with the exclusion from the analysis of people who died in the 3-year period after the care processes were assessed, suggest that the association with higher mortality in those not receiving all eight care processes is not solely due to care processes being suspended for clinical reasons. Furthermore, a sensitivity analysis including all people included in the 2009/2010 NDA and still alive on January 1, 2011 did not significantly alter the fully adjusted results of this analysis (Table S6).

For those with type 2 diabetes, but not type 1 diabetes, there were differences by ethnicity in the association between fewer care processes recorded and higher mortality. Among people with type 2 diabetes, the HR of death from all causes amongst those receiving five or fewer annual care processes was 1.29 (95\% Cl 1.26-1.32) for White ethnicity, 1.13 (95\% Cl 1.03-1.23) for South Asian ethnicity and 1.34 (95\% Cl 1.19-1.52) for Black ethnicity. The lower HR in people of South Asian ethnicity may link to their higher risks of developing type 2 diabetes, but lower subsequent mortality. A study using the clinical practice research datalink cohort reported that the additional risk of dying attributable to diagnosed diabetes was lower in people from South Asian ethnic groups than in those from White ethnic groups, ${ }^{15}$ despite a greater diagnosed incidence of cardiovascular disease. ${ }^{16,17}$ Thus, the smaller additional diabetes-related mortality risk experienced by people from South Asian ethnic groups compared to White ethnic groups may narrow the additional mortality associated with not receiving care processes. Equally, other factors such as health-related behaviours, health beliefs and cultural differences may influence attitudes to healthcare, in particular routine and preventative care, and thereby play a role in explaining this difference.

No previous study has investigated whether the number of recorded care processes is associated with future outcomes in people with diabetes. Nonattendance at clinics and noncompletion of care processes clearly overlap. A recent comprehensive review of the literature on nonattendance at diabetes outpatient appointments ${ }^{18}$ found relationships to both logistical and psychosocial factors. It also found associations with nonattendance at diabetes clinics that were similar to those recognized in other medical specialties, such as young age, social deprivation and smoking. Very few studies of nonattendance at diabetes clinics have investigated subsequent outcomes. ${ }^{19}$ Those that did mostly found associations between infrequent attendance and higher levels of glucose, BMI, blood pressure and lipids, a few studies documented higher emergency hospital use and diabetes-related complications, and just one study, using a composite measure of nonattendance and treatment noncompliance, found higher mortality in people with type 1 diabetes. ${ }^{18,19}$

As compared to the collective results, analysis of the associations between mortality and noncompletion of individual care processes showed variation from higher risk (eg, BMI, cholesterol and foot examinations) to lower risk (eg, blood pressure, smoking enquiry, serum creatinine). Only one individual care process association with mortality differed between type 1 diabetes and type 2 diabetes. Noncompletion of $\mathrm{HbA1c}$ measurement was associated with higher risk in type 1 diabetes but not in type 2 diabetes, perhaps reflecting the greater severity and dominance of hyperglycaemia as a risk factor for complications in type 1 diabetes.

It should be noted that the adjustment of these associations was restricted to age, sex, deprivation and ethnicity as missing data on the risk factors uncovered by the individual care processes hinder more comprehensive adjustments. This means it is plausible that residual confounding and differing risk factor profiles explain these associations. In addition, when carrying out the care processes, previous measurements may influence clinical prioritization, with greater effort being expended on reaching those at previously identified higher risk. It is possible that the proportion of care processes completed is strongly influenced by logistic issues that result in missed appointments, whereas omission of individual items such as weight and surveillance for early complications, may be influenced also by psychosocial factors. Additionally, it may be that some factors recorded as satisfactory and stable at recent visits (eg, HbA1c in people with type 2 diabetes, or blood pressure and kidney function in younger people), are not always repeated, and that a smoking status enquiry may be omitted in long-term nonsmokers, although the primary care pay-for-performance system (Quality and Outcomes Framework) is designed to mitigate against this. Qualitative studies have shown the therapeutic relationship between patient and healthcare professional to be an important determinant of attendance ${ }^{18}$ but the NDA cannot capture this aspect of care.

The present analysis identifies an association between low numbers of annual care processes completed and subsequent 7-year mortality. Therefore, it identifies a group of people who have a higher risk of mortality. But observational analyses cannot establish cause and effect and we cannot exclude residual confounding. One can only speculate on what any mechanism might be. The prominence of respiratory disease among those who died after low rates of care process completion raises one possibility. Respiratory deaths in younger people are predominantly due to pneumonia, for which diabetes is a known risk factor. ${ }^{20}$ In our analysis, we tried to account for known pneumonia risks such as smoking, which was more common in the low care process group, and elevated BMI, but we were not able to include other known factors such as high alcohol intake, poor diet and low physical activity. Conceivably, these unmeasured risks triangulate with the likelihood of missing care processes. Alternatively, individuals more engaged with self-care and lower risk lifestyles may attend clinics more often and be keener to complete all the care processes. Equally, the findings may be due to reverse causality, whereby people with multimorbidities, particularly mental illness, will be less likely to engage with routine follow-up and self-management.

Strengths of the present study include the size of the cohort included in the analysis, covering $76 \%$ of practices in England and Wales, the fact that it is drawn from a comprehensive selection of real-world population-based healthcare records, and the length of the 
follow-up. An important limitation is that neither medication data nor influenza and pneumonia immunizations were available for this analysis, and these could have shed some light on healthcare interactions. The nature of this analysis means that if people have not received a specific care process the risk factor data arising from that process are missing. In this analysis all variables included in the Cox proportional hazard regression models are treated as categorical variables and have a category for "missing" data. Whilst this does not completely eradicate residual confounding due to missing data, it is much reduced. It is not possible to distinguish the separate or joint contributions of inertia from patients or healthcare professionals to undertaking of care processes and therefore the recording of risk factors. To better understand the nature of the associations between the receipt of care processes and disease outcomes and the roles of associations between health beliefs, health behaviours and interactions with healthcare providers requires further qualitative and quantitative work in people with diabetes and their care providers. In addition, the identification of care processes received is limited to a single 15-month period. Variation in interactions with healthcare, and organizational changes to the health service over the follow-up period may have influenced mortality. Data on prescriptions for glucose-lowering drugs were not available for the time period of this analysis. This means that it is not possible to identify whether the associations found in people with type 2 diabetes vary by treatment regimen.

In summary, even when many possible contributory risks for death are taken into account, people with diabetes have a higher mortality risk if their records of routine care indicate several missing annual care processes. Although further evidence is needed on whether efforts to specifically engage this group would yield worthwhile health benefits, health economies should consider how to minimize barriers to receiving the recommended care processes. These observations may be particularly pertinent in contemporary healthcare provision as professionals consider how to organize routine diabetes reviews in the face of the backlog attributable to the direct and indirect effects of COVID-19. It would be all too easy to overlook this high-risk group.

\section{ACKNOWLEDGMENTS}

The authors thank Liz Coyle, University of Glasgow, for her assistance in the preparation of this article. N.H. is currently funded by Diabetes UK and NHS England and NHS Improvement. N.H. received funding from the South Asian Health Foundation for this work. N.S. is supported by the British Heart Foundation Research Excellence Award (RE/18/6/34217).

\section{CONFLICT OF INTERESTS}

Naomi Holman, Bob Young, Naveed Sattar, Kamlesh Khunti, Sarah H. Wild, Edward W. Gregg and Jonathan Valabhji are members of the National Diabetes Audit Research Advisory Group. Naveed Sattar has received grant and personal fees from Boehringer Ingelheim, and personal fees from Amgen, AstraZeneca, Eli Lilly, Merck Sharp \& Dohme, Novartis, Novo Nordisk, Pfizer, and Sanofi outside the submitted work. Kamlesh Khunti has acted as a consultant, speaker or received grants for investigator-initiated studies for Astra Zeneca, Novartis, Novo Nordisk, Sanofi-Aventis, Lilly and Merck Sharp \& Dohme, Boehringer Ingelheim, Bayer, Berlin-Chemie AG/Menarini Group, Janssen, and Napp. Jonathan Valabhji is the National Clinical Director for Diabetes and Obesity at NHS England and NHS Improvement. All other authors declare no relationships or activities that could appear to have influenced the submitted work.

\section{AUTHOR CONTRIBUTIONS}

The study was designed by Roger Gadsby, Bob Young, Naomi Holman and Naveed Sattar. Naomi Holman undertook the statistical analysis. All authors reviewed the methods, assisted in writing the paper, reviewed the final manuscript, and gave approval for publication. Naomi Holman is the guarantor and accepts full responsibility for the work and/or the conduct of the study, had access to the data, and controlled the decision to publish.

\section{PEER REVIEW}

The peer review history for this article is available at https://publons. com/publon/10.1111/dom.14528.

\section{DATA AVAILABILITY STATEMENT}

Information governance rules for the National Diabetes Audit prevent the raw or processed data used in this analysis being made publicly available.

\section{ORCID}

Hermione Price (D) https://orcid.org/0000-0003-3388-0975

Richard I. G. Holt (D) https://orcid.org/0000-0001-8911-6744

Kamlesh Khunti (D) https://orcid.org/0000-0003-2343-7099

Naveed Sattar (D) https://orcid.org/0000-0002-1604-2593

\section{REFERENCES}

1. UK Prospective Diabetes Study (UKPDS) Group. Effect of intensive blood-glucose control with metformin on complications in overweight patients with type 2 diabetes (UKPDS 34). UK Prospective Diabetes Study (UKPDS) Group. Lancet. 1998;352(9131):854-865. https://doi. org/10.1016/S0140-6736(98)07037-8

2. Cholesterol Treatment Trialists' (CTT) Collaborators, Mihaylova B, Emberson J, et al. The effects of lowering LDL cholesterol with statin therapy in people at low risk of vascular disease: meta-analysis of individual data from 27 randomised trials. Lancet. 2012;380(9841): 581-590. https://doi.org/10.1016/S0140-6736(12)60367-5

3. de Boer IH, Bangalore S, Benetos A, et al. Diabetes and hypertension: a position statement by the American Diabetes Association. Diabetes Care. 2017;40(9):1273-1284. https://doi.org/10.2337/ dci17-0026

4. National Institute for Health and Care Excellence. Type 1 diabetes in adults: diagnosis and management, NICE guideline (NG17). https:// www.nice.org.uk/guidance/ng17. Accessed March 20, 2019.

5. National Institute for Health and Care Excellence. Type 2 diabetes in adults: management NICE guideline. https://www.nice.org.uk/ guidance/ng28. Accessed August 16, 2019.

6. International Diabetes Federation. IDF Global Guideline for Type 2 Diabetes. https://www.idf.org/e-library/guidelines/79-globalguideline-for-type-2-diabetes. Published 2017. Accessed March 25, 2019 
7. American Diabetes Association. 3. Comprehensive medical evaluation and assessment of comorbidities. Diabetes Care. 2017;40(suppl 1): S25-S32. https://doi.org/10.2337/dc17-S006

8. NHS Digital. Quality Outcomes Framework (QOF). 2020. https:// digital.nhs.uk/data-and-information/data-tools-and-services/dataservices/general-practice-data-hub/quality-outcomes-frameworkqof. Accessed January 17, 2020.

9. Holman N, Knighton P, Wild SH, et al. Cohort profile: National Diabetes Audit for England and Wales. Diabet Med. 2021;38:e14616. https://doi.org/10.1111/dme.14616

10. NHS Digital. National Diabetes Audit - 2009-10, Audit analysis https://digital.nhs.uk/data-and-information/publications/statistical/ national-diabetes-audit/national-diabetes-audit-2009-10-auditanalysis. Accessed August 5, 2021.

11. Office for National Statistics. Index of Multiple Deprivation (IMD) 2007. https://data.gov.uk/dataset/bdc1e1a5-aaf3-4f5a-9988-82a11e341eb8/ index-of-multiple-deprivation-imd-2007. Accessed April 8, 2021.

12. Levey AS, Coresh J, Greene T, et al. Using standardized serum creatinine values in the modification of diet in renal disease study equation for estimating glomerular filtration rate. Ann Intern Med. 2006;145(4): 247-254. https://doi.org/10.7326/0003-4819-145-4-20060815000004

13. Breslow NE, Day NE. Statistical methods in cancer research. Volume II-the design and analysis of cohort studies. IARC Sci Publ. 1987;82: 1-406. http://publications.iarc.fr/Book-And-Report-Series/larcScientific-Publications/Statistical-Methods-In-Cancer-ResearchVolume-II-The-Design-And-Analysis-Of-Cohort-Studies-1986. Accessed March 22, 2019.

14. Seidu S, Davies MJ, Farooqi A, Khunti K. Integrated primary care: is this the solution to the diabetes epidemic? Diabet Med. 2017;34(6): 748-750. https://doi.org/10.1111/dme.13348

15. Wright AK, Kontopantelis E, Emsley R, et al. Life expectancy and cause-specific mortality in type 2 diabetes: a population-based cohort study quantifying relationships in ethnic subgroups. Diabetes Care. 2017;40(3):338-345. https://doi.org/10.2337/dc16-1616
16. Malik MO, Govan L, Petrie JR, et al. Ethnicity and risk of cardiovascular disease (CVD): 4.8 year follow-up of patients with type 2 diabetes living in Scotland. Diabetologia. 2015;58(4):716-725. https://doi.org/ 10.1007/s00125-015-3492-0

17. Tillin T, Hughes AD, Mayet J, et al. The relationship between metabolic risk factors and incident cardiovascular disease in Europeans, South Asians, and African Caribbeans: SABRE (Southall and Brent Revisited)-a prospective population-based study. J Am Coll Cardiol. 2013;61(17):1777-1786. https://doi.org/10.1016/j.jacc.2012.12.046

18. Brewster S, Bartholomew J, Holt RIG, Price H. Non-attendance at diabetes outpatient appointments: a systematic review. Diabet Med. 2020;37(9):1427-1442. https://doi.org/10.1111/dme.14241

19. Currie CJ, Peyrot M, Morgan CL, et al. The impact of treatment noncompliance on mortality in people with type 1 diabetes. J Diabetes Complications. 2013;27(3):219-223. https://doi.org/10.1016/j. jdiacomp.2012.10.006

20. Torres A, Peetermans WE, Viegi G, Blasi F. Risk factors for community-acquired pneumonia in adults in Europe: a literature review. Thorax. 2013;68(11):1057-1065. https://doi.org/10.1136/ thoraxjnl-2013-204282

\section{SUPPORTING INFORMATION}

Additional supporting information may be found in the online version of the article at the publisher's website.

How to cite this article: Holman N, Knighton P, O'Keefe J, et al. Completion of annual diabetes care processes and mortality: A cohort study using the National Diabetes Audit for England and Wales. Diabetes Obes Metab. 2021;23(12): 2728-2740. doi:10.1111/dom.14528 